\title{
L'aventure didactique du Télémaque de Fénelon en Bulgarie
}

Dimitar Vesselinov

\section{(2) OpenEdition \\ 12 Journals}

\section{Édition électronique}

URL : https://journals.openedition.org/dhfles/1069

DOI : 10.4000/dhfles.1069

ISSN : 2221-4038

Éditeur

Société Internationale pour l'Histoire du Français Langue Étrangère ou Seconde

Édition imprimée

Date de publication : 1 décembre 2005

Pagination : 27-39

ISSN : 0992-7654

\section{Référence électronique}

Dimitar Vesselinov, «L'aventure didactique du Télémaque de Fénelon en Bulgarie », Documents pour

I'histoire du français langue étrangère ou seconde [En ligne], 35 | 2005, mis en ligne le 29 mai 2011, consulté le 27 mai 2021. URL : http://journals.openedition.org/dhfles/1069 ; DOI : https://doi.org/ 10.4000/dhfles.1069

Ce document a été généré automatiquement le 27 mai 2021.

(C) SIHFLES 


\title{
L'aventure didactique du Télémaque de Fénelon en Bulgarie
}

\author{
Dimitar Vesselinov
}

1 Cette contribution à l'histoire européenne des Aventures de Télémaque est inspirée de l'étude de Lioubomir Vankov sur l'influence idéologique et culturelle du roman de Fénelon dans les Balkans. Bien qu'elles constituent une masse de lecteurs fort disparates, les différentes ethnies de la péninsule, malgré les difficultés provoquées par la situation politique et religieuse instaurée dans l'Empire ottoman, suivent avec intérêt les idées du siècle des Lumières et observent avec passion et espoir les transformations paneuropéennes engendrées par la Révolution française et propagées par les guerres napoléoniennes. Mon objectif est, sur la base chronologique de la réception balkanique du roman, de retracer l'ampleur de l'accueil didactique de Télémaque en Bulgarie au XIXe siècle, un accueil qui dépasse largement la visée pédagogique de Fénelon, son impact culturel et son influence idéologique.

C'est à l'époque de la Renaissance nationale, soit de 1762 à 1878, que le chef-d'œuvre de Fénelon se répand en Bulgarie sous sa forme originale ou traduite, pour y jouir d'une notoriété et d'un prestige extraordinaires. Très vite, par sa dimension interculturelle reliant l'Antiquité à la Modernité, l'Orient hellénique à l'Occident plus ou moins latinisé, le rêve du souverain éclairé aux idées réformatrices, les postulats éducatifs d'inspiration humaniste à la pratique de l'enseignement du français et de la civilisation européenne, LesAventures de Télémaque deviennent le symbole des idées libérales qui formeront la nouvelle élite intellectuelle bulgare.

3 L'hypothèse que je développerai ici, toutefois, est que la grande popularité des Aventures de Télémaque en Bulgarie est avant tout due à l'usage didactique du roman établi de bonne heure sur toute la péninsule. Ce postulat ne met pas en doute le rôle que joue la légende hellénique dans la réception favorable des pérégrinations de Télémaque en Thrace ancienne, mais je pense que le potentiel didactique du roman reste dominant dans cet étrange échange interculturel entre le Levant et l'Occident, les Balkans et l'Europe francophone. 
4 Dans les écoles bulgares, grecques, roumaines, serbes, turques... partout on introduit avec plus ou moins de ferveur et d'enthousiasme l'enseignement des langues étrangères par la lecture et la traduction des Aventures de Télémaque dans la langue maternelle, les accompagnant de brefs commentaires grammaticaux. Dans certains pays balkaniques, cette pratique est poussée à l'extrême : les pédagogues bulgares, par exemple, enseignent non seulement le français mais le grec, l'italien, le roumain et le turc à l'aide du Télémaque. Le livre acquiert ainsi une place spéciale dans les foyers bulgares à côté de l'Évangile.

5 L'œuvre de Fénelon pénètre dans la péninsule balkanique directement sous sa forme originale dès le début du XVIII ${ }^{e}$ siècle. Selon Lioubomir Vankov, la traduction italienne paraît, à un moment donné, être la seule concurrente de la version française. Mais Télémaque devient vraiment populaire grâce aux nombreuses traductions qu'on en donne dans les langues balkaniques, surtout au début et dans la première moitié du XIXe siècle. Entre-temps on lit l'original français qui se révèle aux admirateurs de la culture française à mesure que les Balkans s'ouvrent à l'influence de la modernité européenne. La réception de Télémaque en Bulgarieest facilitée par «le fait que les peuples de la péninsule ne font pas connaissance du roman isolément et chacun pour son propre compte mais qu'ils s'initient mutuellement à la compréhension et à l'amour du chef-d'œuvre de Fénelon. $»^{1}$

6 La Grèce est le premier pays des Balkans à prendre connaissance du livré de Fénelon au début du XVIII ${ }^{e}$ siècle. L'analyse des relations culturelles franco-grecques démontre que l'auteur du Télémaque est l'écrivain le mieux connu dans ce pays tandis que son roman s'avère être parmi les œuvres littéraires ayant acquis un immense succès. La première traduction de Télémaque en grec moderne date de 1742, ce qui ouvre la voie à sa diffusion dans les Balkans. Le traducteur est Athanassios Skiadas, spécialiste en langues classiques, qui étudie le droit et les sciences économiques en Italie. Devenu professeur de grec à Moscou il s'attache aux idées de Fénelon, ce qui lui permet de s'inscrire dans l'histoire littéraire avec ses deux traductions de Télémaque, hautement appréciées de ses contemporains : l'une en russe et l'autre en grec moderne. La seconde traduction de Télémaque en grec moderne paraît en 1801. Le traducteur, Dimitrios Govdelas, fait ses études dans des universités allemandes et hongroises avant de devenir directeur de l'Ecole centrale grecque de Jassy en Moldavie. Il dédie sa traduction «aux enfants grecs » qui découvriront à travers le texte fénelonien de nombreuses réminiscences historiques qui seront utiles à leur éducation : il ne faut pas oublier le fait que le célèbre auteur français suit les pas des anciens Grecs pour créer son œuvre. Konstantinos Ikonomos (1780-1875) est le troisième traducteur grec de Télémaque. Il est né en Thessalie où il reçoit une bonne éducation et apprend la langue française. En 1808 il devient professeur de philologie et de rhétorique à Smyrne où il s'adonne à la traduction. Souhaitant rendre un nouvel éclat au roman de Fénelon, il donne une version grecque des Aventures de Télémaque en vers hexamétriques, considérant l'écrivain français comme successeur poétique d'Homère et de Virgile.

7 Le roman de Fénelon commence à être connu en Valachie et en Moldavie (les deux anciennes principautés danubiennes qui donnèrent naissance à la Roumanie) entre les années soixante et soixante-dix du XVIII e siècle. C'est le premier livre que les enfants de l'aristocratie reçoivent de leurs précepteurs et il est naturel que la traduction de Télémaque soit empreinte du souci didactique : la plus ancienne traduction en roumain est une version manuscrite anonyme des cinq premiers livres du roman, faite en 1772 
(Eliade 1898). En 1807 Petru Major (1760-1821), professeur en Transylvanie, traduit de l'italien Les Aventures de Télémaque. Il prend connaissance du roman à Rome où il fait ses études en philosophie et en théologie. La première traduction directe du français est faite en 1831 par Grigorij Plechoianu. Le texte est rédigé en cyrillique, typique de cette époque de l'histoire roumaine. Il est fort possible que cette traduction soit utilisée dans les cours de français du lycée «Saint Save » de Bucarest (Urechia 1892 : 13)². La seconde traduction du français est l'œuvre d'A. I. Cretescu et paraît en 1852 à Bucarest. Cet intellectuel roumain est frappé par l'harmonie de la langue fénelonienne qui présente des opportunités incontestables pour l'apprentissage de la langue française.

Les Serbes découvrent Fénelon et son Télémaque dans le dernier quart du XVIII ${ }^{\mathrm{e}}$ siècle de manière indirecte à travers les écrits de Dossitej Obradovitch qui recommande « aux érudits serbes » de traduire ce roman, considérant le texte de Fénelon comme « l'un des meilleurs outils pour rendre la culture française plus proche des Serbes » (Stikič 2003: 66). Gligorié Terlaïtch (1766-1811) laisse une traduction manuscrite non datée de Télémaque. Ce n'est qu'en 1814 qu'apparaît la première traduction imprimée du roman, due à Stéfan Zivkovitch et faite à partir de l'édition parisienne des Aventures de Télémaque. À la différence des autres pays balkaniques, le Télémaque serbe n'arrive pas à jouer le même rôle dans les écoles au moment où le français devient une matière scolaire.

9 La version originale du roman de Fénelon parvient en Bulgarie dans le second tiers du $\mathrm{XIX}^{\mathrm{e}}$ siècle, vraisemblablement entre les années trente et quarante, tandis que les traductions grecques, roumaines et serbes sont déjà bien connues. La traduction serbe de 1814 jouit d'une popularité dans les villes de Bulgarie du Nord, les versions grecques sont introduites par l'élite hellénophone dans les grands centres éducatifs du pays, tandis que l'émigration bulgare en Valachie et en Moldavie utilise, selon les cas, l'une des deux traductions roumaines. Dans les écoles bulgares de Bessarabie, on a aussi accès à la version russe.

10 Cet intérêt pour le français et la France coïncide avec l'écho ressenti en Europe par le voyage dans les Balkans d'Adolphe Blanqui en 1841 et la publication de ses notes de voyage en 1842. À cela s'ajoutent encore les programmes de pénétration culturelle au Levant du baron Bilingue, diplomate français en poste à Rome et de Cyprien Robert, professeur au Collège de France. Dans ses dépêches d'avril 1842 le baron Bilingue insiste sur l'intensification de l'enseignement du français à l'Est par l'intermédiaire des ordres religieux, tandis que Cyprien Robert propose dans la Revue des deux mondes de la même année, l'ouverture d'imprimeries slaves en France pour le renforcement de l'influence française dans les Balkans. Ainsi, après sa première audience chez Guizot, le jeune Bulgare Alexandre Exarque lance-t-il plusieurs initiatives pour la promotion du français et de la France en Bulgarie. Le début des années quarante du XIX ${ }^{\mathrm{e}}$ siècle est donc marqué par une atmosphère favorable à l'expansion du français dans la péninsule balkanique. L'intérêt des Bulgares à l'égard de la culture et de la science françaises, considérées comme condition nécessaire au développement rapide de leur renaissance spirituelle, est manifeste.

11 Tout ce contexte crée une atmosphère favorable à l'apparition de la première traduction slavo-bulgare de Télémaque en 1845. Le traducteur, P. G. Pipérov achève son travail en 1844 mais le livre sort de l'imprimerie une année plus tard. Bien que sa traduction ne soit pas intégrale - elle se limite aux six premiers livres du roman - elle demeure un phénomène peu ordinaire dans l'histoire culturelle de la Bulgarie. Le 
Télémaque de Fénelon s'avère être la première création littéraire du monde occidental publiée en bulgare à l'époque de la Renaissance nationale et conservera bien longtemps sa position d'événement culturel sans réelle concurrence. À une époque où le goût esthétique et la valeur artistique sont aléatoires, le choix du roman fénelonien est une trouvaille ingénieuse du traducteur qui va aussitôt jouir d'une popularité bien méritée. Arrêtant son regard sur Télémaque, Pipérov manifeste une intuition culturelle et pédagogique assez rare pour son temps. À la différence de ceux qui s'intéressent aux auteurs en vogue que l'histoire qualifiera d'éphémères et à la différence d'érudits qui optent pour des adaptations de l'original, il reste quant à lui, fidèle au texte. Pipérov ne veut pas abaisser Télémaque au niveau esthétique du lecteur moyen mais au contraire, essaie d'amener la réception littéraire du lecteur bulgare à la hauteur de l'œuvre française. Le traducteur partage l'enthousiasme éclairé des éveilleurs de la conscience nationale qui considèrent que le premier pas vers l'indépendance reste toujours l'éducation rationnelle. L'élan culturel, à une époque où les Bulgares commencent à s'auto évaluer par rapport à leurs voisins et aux autres nations européennes, serait certainement incomplet si l'on restait en dehors de la communauté bénéficiant dans sa langue des valeurs véhiculées par les chefs-d'œuvre de la civilisation dont fait partie Télémaque.

P. G. Pipérov est né à Svichtov. Il fait ses études dans sa ville natale où l'apprentissage du grec, du français et de l'allemand jouit d'une grande popularité. Probablement Pipérov continue son éducation dans une université étrangère avant de s'installer à Bucarest où il accomplit sa traduction de Télémaque en 1844. Après un voyage en Allemagne et un arrêt à Leipzig en 1846, il continue son activité dans la capitale roumaine. Mais à sa réputation de négociant s'est ajoutée la renommée d'un homme de lettres hautement apprécié de l'élite bulgare. Le futur consul russe et lexicographe chevronné, Najden Guérov, le place parmi les éminents littérateurs bulgares. Cette notoriété du traducteur est due aussi à sa bonne connaissance du slavo-bulgare qui le range parmi les intellectuels conservateurs. De fait, avec le choix du registre langagier de sa traduction, il ne s'oppose pas à l'utilisation du bulgare moderne mais préconise l'utilité pédagogique du slavo-bulgare qui est la langue ecclésiastique. Vu la tradition littéraire des années quarante du XIXe siècle, Pipérov opte pour une variante classique de la langue nationale tout en étant conscient de ses insuffisances. Il est persuadé que la perfection de l'original ne pourrait être reproduite qu'au moyen du slavo-bulgare et il se permet parfois d'inventer ou d'adapter des termes afin de mieux traduire la pensée fénelonienne. Pipérov fait preuve d'une bonne connaissance du français et d'une habileté à trouver des équivalents ingénieux pour traduire les passages difficiles du Télémaque en slavo-bulgare, mais cela ne peut pas neutraliser le sentiment de vétusté que revêt sa traduction. C'est sans doute la raison pour laquelle plusieurs enseignants se mettent à faire leurs propres traductions ou améliorations de Télémaque pour répondre aux exigences modernes de l'enseignement du français dispensé dans les écoles bulgares. Le texte de Pipérov est fidèle à l'original et trouve une application didactique assez fructueuse dans l'enseignement du français, mais il n'arrive pas à accomplir complètement son rôle culturel et littéraire.

13 Voilà pourquoi, une vingtaine d'années plus tard, en 1873, paraît une seconde traduction des Aventures de Télémaque faite par Nikola Michaïlovski. Le traducteur est un érudit bulgare aux intérêts et aux goûts littéraires. Il arrive à reproduire l'effet stylistique et poétique du texte français, ce qui met davantage en valeur l'orientation didactico-pédagogique du roman et assure sa réception positive par les apprenants 
bulgares. Le traducteur est conscient de l'impact dont jouit Télémaque sur le public balkanique et se borne à vanter l'excellence fénelonienne, non pas comme une fin en soi, mais dans l'optique de faire valoir aussi la richesse de la langue bulgare qui a rendu possible une telle initiative. La traduction intégrale des Aventures de Télémaque, dans la stratégie apologétique de Nikola Michaïlovski, est une preuve irréfutable de la subtilité que le bulgare moderne a atteint dans son développement intensif des dernières années. Le traducteur n'a pas besoin de motiver son choix, vu la renommée dont bénéficie déjà le roman, mais il recourt aux classiques français tels que Boileau et Voltaire pour accentuer les qualités littéraires de l'œuvre fénelonienne. À la différence de son prédécesseur Pipérov, qui ne voyait en Télémaque qu'enjeux pédagogiques et culturels, Nikola Michaïlovski sait apprécier la poétique du roman et l'harmonie de la langue française. Plus de trente ans se sont écoulés depuis la première traduction du Télémaque et cela se constate à l'évolution du paradigme didactico-réceptif. Le roman n'est plus un simple moyen d'acquisition de la langue française mais un échantillon littéraire à imiter et les chrestomathies nouvellement sorties dans les pays balkaniques en attestent. La qualité de la traduction de Michaïlovski est impressionnante. Le registre utilisé du bulgare est très proche de la langue parlée, ce qui donne une actualité dynamique au texte traduit. Mises à part certaines particularités de l'orthographe non codifiée, la version bulgare de Michaïlovski n'empêche pas le lecteur d'avoir une perception directe du texte fénelonien. Cette seconde traduction du Télémaque permet au roman, non seulement d'atteindre le public bulgare dans son intégralité mais aussi de mettre en relief l'art narratif et la perfection du style fénelonien.

14 À la différence de la tradition européenne selon laquelle Les Aventures de Télémaque sont introduites dans l'enseignement de la langue française sous forme d'édition bilingue, en Bulgarie on utilise deux corps livresques: le texte original et sa traduction bulgare officielle ou didactiquement adaptée (faite dans la majorité des cas par l'enseignant luimême). En 1836 Néofite Bozvéli apporte à Svishtov la version serbe de Télémaque afin de l'intégrer à son enseignement. En 1840 à Ohkrid, Dimitar Miladinov fait traduire à ses élèves des pages du roman fénelonien. Pendant l'année scolaire 1848-49 à l'école de Pléven (Plévna), Emanouil Vaskidovitch utilise largement dans ses cours de français, des extraits de Télémaque, choisis en fonction de leur portée pédagogique et soigneusement traduits peu de temps auparavant. La même année, Sava Dobroplodni enseigne le français à Choumen (Choumla) d'après Les Aventures de Télémaque en faisant recopier à ses élèves différents passages de sa traduction manuscrite (1849-1859). Le texte de Fénelon est également utilisé dans les cours de français dispensés à l'école de Koprivtchitza en 1864-1865 et à l'école de Sofia en 1865-1866. Le patriarche de la littérature bulgare, Ivan Vazov, apprend le français en traduisant tout le Télémaque à l'aide du dictionnaire. Dès le début des années soixante-dix du XIXe siècle, dans les programmes scolaires de l'école bulgare de Constantinople, Les Aventures de Télémaque occupent une place centrale d'autant plus qu'il y a déjà d'excellentes traductions turques, grecques et russes dans les librairies de la capitale ottomane. Des extraits du roman fénelonien sont introduits à des fins didactiques dans la chrestomathie de Dobri Vojnikov (1860) et dans le manuel de stylistique élémentaire de l'ancien élève de la Sorbonne Todor Chichkov (1873), où les auteurs réservent une place honorifique à Fénelon. La popularité de Fénelon est si grande que les éditions françaises de Télémaque sont connues, non seulement des apprenants bulgares mais aussi de leurs 
parents qui les installent progressivement sur les étagères domestiques traditionnellement réservées aux livres religieux obligatoires.

À partir des années soixante du XIXe siècle, Télémaque est utilisé même dans l'enseignement de la langue turque dispensé à Plovdiv par Joakim Grouev. D'après le pédagogue Alexandre Teodorov Jivkov, la langue fénelonienne doit servir d'exemple au développement de l'éloquence des apprenants bulgares, y compris dans les cours de langue maternelle. Des exemples tirés des écrits littéraires de cette époque attestent de l'utilisation des phrases de Télémaque dans les conversations quotidiennes «à la franga » (à la française) entre des " érudits autochtones ». Hariton Guénadiev souligne que jusqu'au milieu du XIXe siècle, les Bulgares qui se mettent à étudier la langue française même à des fins pratiques, recourent à la comparaison des textes français de l'Evangile et du Télémaque fénelonien avec leurs versions bulgares. Rien d'étonnant donc qu'à la fin du XIXe siècle, bon nombre de vieux érudits écrivant et parlant le français n'aient eu d'autre support pédagogique que Les Aventures de Télémaque.

Pour les apprenants bulgares, le roman de Fénelon joue le rôle non seulement de manuel de français, mais de jalon moral leur indiquant l'exemple suivi par les nations occidentales qui ont joui de l'expansion des sciences et des arts. Il leur recommande le respect envers la lecture et les livres comme le fait Télémaque dont l'esprit infatigable ne cesse de chercher de nouvelles connaissances : « heureux ceux qui se divertissent en s'instruisant et qui se plaisent à cultiver leur esprit par les sciences ", " heureux ceux qui aiment à lire et qui ne sont point [...] privés de la lecture ». Et ce n'est pas tout. Pour les apprenants bulgares, Télémaque requiert un statut symbolique : la lecture du fameux roman de Fénelon est assez particulière pour la jeunesse bulgare, différente de toute lecture occidentale ou d'Europe centrale, parce qu'on y découvre l'esprit de l'ancienne Thrace, qu'on y entend la musique d'Orphée, qu'on y reconnaît sa patrie dans l'image de l'état mythique de Bétique et qu'on y capte le moindre appel à la liberté en dépit de la prudence extrême du rationalisme fénelonien.

Le déclin de l'époque didactique de Télémaque survient en Bulgarie avec l'apparition du manuel de français de Spas Zafirov (1862) rédigé dans l'esprit ollendorffien et surtout avec l'impression du dictionnaire français-bulgare et bulgare-français d'Ivan Bogorov (1869). L'édition de ces deux excellents supports pédagogiques annonce la fin du phénomène remarquable, dans l'histoire culturelle de la Bulgarie, qu'est l'œuvre fénelonienne. Les versions françaises du Télémaque, accompagnées ou non de traductions bulgares, commencent à tomber dans l'oubli vers la fin des années soixante-dix du $\mathrm{XIX}^{\mathrm{e}}$ siècle et si l'on continue de les trouver dans quelques bibliothèques privées, on les considère plutôt comme des curiosités de bibliophiles, souvenirs d'une époque déjà révolue et non plus comme outils d'apprentissage univoque du français. 


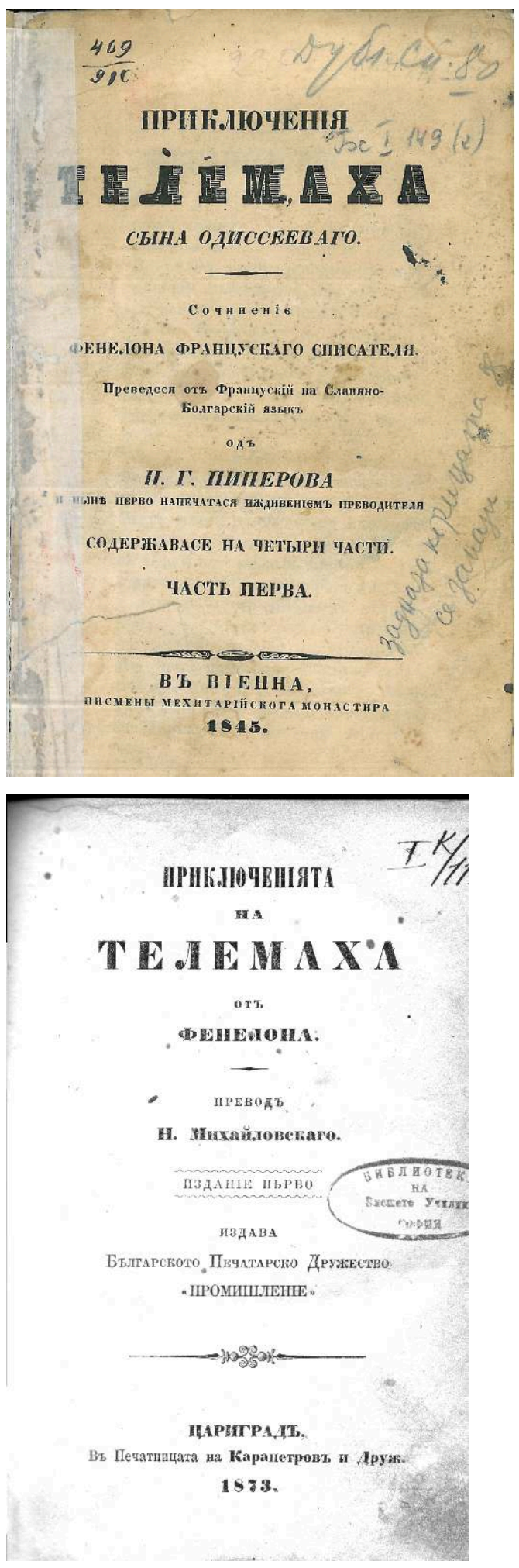




\section{BIBLIOGRAPHIE}

\section{Traductions de Télémaque}

ФЕНЕЛОВ, ФРАНСОА. ПРИКЛЮЧЕНИЯ ТЕЛЕМАХА, СИНА ОДИССЕВАГО. СОЧИНЕНИЕ ФРАНЦУЗСКАГО

СПИСАТЕЛЯ. ПРЕВЕДЕ СЯ ОТ ФРАНЦУЗСКИЙ НА СЛАВЯНО-БОЛГАРСКИЙ ЯЗЫК ОТ П. Г. ПИПЕРОВ И НИНЕ ПЕРВО НАПЕЧАТА СЯ С ИЖДИВЕНИЕМ ПРЕВОДИТЕЛЯ. ВИЕНА, ПИСМЕНИ МЕХИТАРИЙСКОГА МОНАСТИРА, 1845,148 С.

МИХАЙЛОВСКИ, Н. ПРИКЛЮЧЕНИЯТА НА ТЕЛЕМАХА ОТ ФЕНЕЛОНА. ПРЕВОД Н. МИХАЙЛОВСКАГО. ИЗДАНИЕ ПЪРВО.ИЗДАВА БЪЛГАРСКОТО ПЕЧАТАРСКО ДРУЖЕСТВО « ПРОМИШЛЕНИЕ », ЦАРИГРАД, $1873,402,6 \mathrm{C}$.

\section{Critique}

ВАНКОВ, Л. ФЕНЕЛОНОВИЯТ ТЕЛЕМАХ В БАЛКАНСКИТЕ СТРАНИ. // ГОДИШНИК НА УНИВЕРСИТЕТА СВ. КЛИМЕНТ ОХРИДСКИ - СОФИЯ, ФИЛОЛОГИЧЕСКИ ФАКУЛТЕТ. ТОМ XL, 1943/44, С. 98.

ELIADE, P. 1898. Del'influence françaisesurl'espritpublicenRoumanie. Les origines. Paris.

URECHIA, V. A. 1892. Istoria scolelor de la 1800-1864. T. II, Bucaresci.

STIKIČ, B. 2003. « La réception et l'utilisation des Aventures de Télémaque en Serbie ». Documents 31 , décembre.

\section{NOTES}

1. ВАНКОВ, Л. ФЕНЕЛОНОВИЯТ ТЕЛЕМАХ В БАЛКАНСКИТЕ СТРАНИ. // ГОДИШНИК НА УНИВЕРСИТЕТА

СВ. КЛИМЕНТ ОХРИДСКИ - СОФИЯ, ФИЛОЛОГИЧЕСКИ ФАКУЛТЕТ. ТОМ XL, 1943/44, С. 98.

2. Le programme scolaire prévoit pour l'année 1837-38 la traduction de Télémaque en classe de seconde.

\section{RÉSUMÉS}

L'hypothèse de l'auteur est que la grande popularité des Aventures de Télémaque en Bulgarie est due à l'usage didactique du roman qui reste dominant dans cet étrange échange culturel entre le Levant et l'Occident, les Balkans et l'Europe francophone. La première traduction est faite en bulgare classique (Pipérov 1845) tandis que la seconde est réalisée en bulgare moderne (Michaïlovski 1873). Le texte de Pipérov est fidèle à l'original et trouve une application didactique assez fructueuse dans l'enseignement du français, mais il n'arrive pas à accomplir complètement son rôle culturel et littéraire. La traduction de Michaïlovski permet au roman, non seulement d'atteindre le public bulgare dans son intégralité mais aussi de mettre en relief l'art narratif et la perfection du style fénelonien. À la différence de la tradition européenne selon laquelle Les Aventures de Télémaque sont introduites dans l'enseignement de la langue française sous forme d'édition bilingue, en Bulgarie on utilise deux corps livresques : le texte original et sa traduction 
bulgare officielle ou didactiquement adaptée (faite dans la majorité des cas par l'enseignant luimême).

The author's assumption is that the great popularity of the Aventures de Télémaque in Bulgaria is due to the didactic use of the novel - which remains dominant in this strange cultural exchange between the Orient and Occident, the Balkans and French-speaking Europe. Pipérov's 1845 work is a translation into classical Bulgarian, while Michaïlovski's 1873 translation renders the text into modern Bulgarian.Pipérov's text is faithful to the original and finds a rather profitable didactic application in the teaching of French, but it does not completely fulfill its cultural and literary role. Michaillovsky's translation, on the other hand, allows the novel not only to reach the larger Bulgarian public, but also to highlight the narrative art and perfection of Fénelon's style. While in the European tradition Télémaque tends to be introduced into French language teaching in the form of a single bilingual edition, in Bulgaria two separate books are used: the original text and its official or didactically-adapted Bulgarian version (translated in the majority of cases by the teachers themselves).

INDEX

Mots-clés : Bulgarie, Télémaque, didactique, réception

Keywords : Bulgaria, Telemaque, didactic, reception

\section{AUTEUR}

\section{DIMITAR VESSELINOV}

Université de Sofia 